\title{
Muon track reconstruction and veto performance with D-Egg sensor for IceCube-Gen2
}

\section{The IceCube Gen2 Collaboration}

http://icecube.wisc.edu/collaboration/authors/icrc17_gen2

E-mail: achim.stoessleicecube.wisc.edu

\begin{abstract}
The planned extension of IceCube, IceCube-Gen2, a cubic-kilometer sized neutrino observatory, aims at increasing the rate of observed astrophysical neutrinos by up to a factor of 10 . The discovery of a high energy neutrino point source is one of its primary science goals. Improving the sensitivity of the individual modules is a necessity to achieve the desired design goal of IceCube-Gen2. A way of improving their sensitivity is the increase of photocathode area. The proposed module called the D-Egg will utilize two 8" Hamamatsu R5912-100 photomultiplier tubes (PMTs), with one facing upwards and one downwards. These PMTs have an increased quantum efficiency and their sensitivity is comparable to the 10" PMT used by IceCube. This essentially leads to an increase in sensitivity by almost a factor of 2 with a full $4 \pi$ solid angle acceptance. A simulation study is presented that indicates improvement in angular resolution using current muon reconstruction techniques due to the new sensor design. Since the proposed module is equipped with an upward facing PMT, further emphasis will be set on the development of new reconstruction techniques that exploit this geometry, as well as an improvement of veto probability for incoming muon tracks, which is crucial for neutrino astronomy in the Southern sky.
\end{abstract}

Corresponding author: A. Stoess1 ${ }^{* 1}$

${ }^{1}$ International Center for Hadron Astrophysics, Graduate School of Science,

Chiba University 1-33, Yayoi-cho, Inage-ku, Chiba-shi, Chiba, 263-8522 Japan

35th International Cosmic Ray Conference - ICRC2017

10-20 July, 2017

Bexco, Busan, Korea

${ }^{*}$ Speaker. 


\section{IceCube Gen2}

The IceCube neutrino observatory at the geographic South Pole is a cubic kilometer array of photosensors which is able to detect the faint Cherenkov light produced by secondaries from interactions of neutrinos with the glacial ice [1]. So far, the experiment has yielded a plethora of science results, among them the discovery of a neutrino flux most likely of extraterrestrial origin [2]. After 6 years of data-taking, with the completed detector, a precise measurement of the extraterrestrial neutrino flux is still limited by statistics. To overcome the statistical limitations and to improve the effective area for neutrino events in the energy regime beyond $10 \mathrm{PeV}$ as well as the point source sensitivity, an extension of the IceCube array has been proposed [3]. The proposed geometry for IceCube-Gen 2 considered in this work is shown in Figure 1. The geometry shows a larger extension in the x-y plane than in depth. It is optimized for the reconstruction of horizontal muon tracks, since these have the highest contribution to the point-source sensitivity [4].

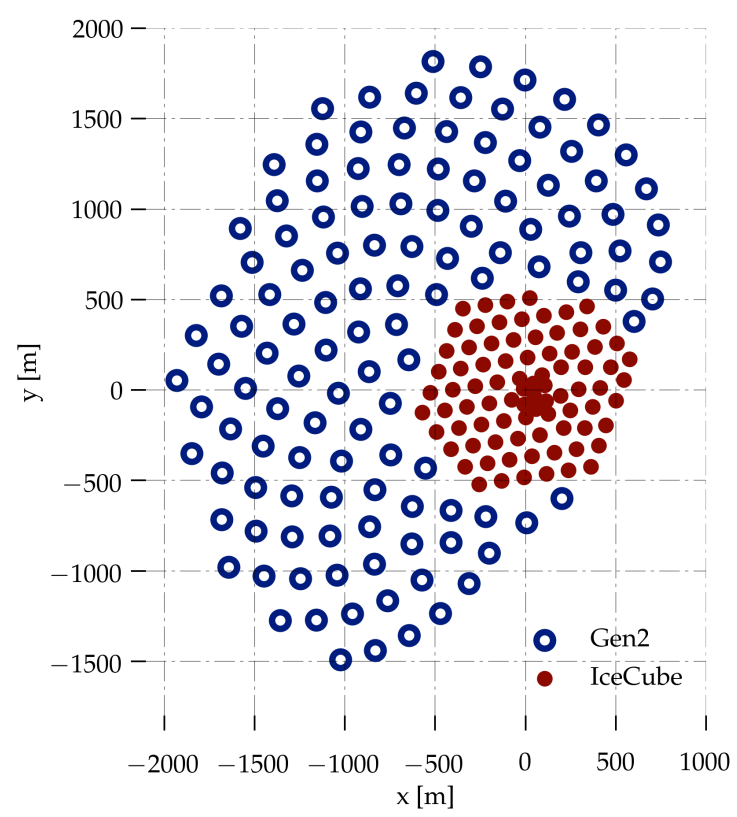

Figure 1: A proposed geometry for IceCube-Gen2 which is used for this study. In addition to the 86 strings of IceCube, which can be seen as the hexagonal shape marked with the red dots, 120 new strings with each 80 sensors are arranged in a complex grid geometry to optimize the veto power for incoming muon tracks. The average distance between the new strings is about $240 \mathrm{~m}$ with a vertical spacing between individual sensors of $17 \mathrm{~m}$. The extension of IceCube to larger positive $\mathrm{x}$-values is prohibited due to the runway of the South Pole Station.

\section{The D-Egg sensor for Gen2}

Several different sensor designs for IceCube-Gen2 are under investigation. However relevant for this study are the following two proposed designs:

- The PDOM [5], which is basically the same design as the IceCube optical sensor [6], however with a PMT with a higher quantum efficiency. It features a single 10" PMT which is facing downwards and a improved readout.

- The D-Egg [7], which follows the design of the PDOM, however includes another PMT facing upwards. The PMTs are 8", so the total diameter of the D-Egg is slightly smaller than the PDOM and it has about 1.48 more photocathode area than the PDOM for a Cherenkov weighted spectrum. 
Due to high drill costs at the South Pole, it is desirable to deploy sensors with a large photocathode area to keep the cost for the average $\mathrm{cm}^{2}$ photocathode as low as possible. The high drill costs can be reduced by drilling holes with a smaller diameter. As the diameter of the D-Egg is $10 \%$ smaller than the diameter of the PDOM, about $20 \%$ of the fuel cost can be saved during deployment. A graphic of the D-Egg with its dimensions is shown in Figure 2. The two Hammaatsu R5912-100 high quantum efficiency PMTs are enclosed in a highly transparent glass housing, which is optimized for transparency in the near ultraviolet. The high voltage for the PMTs is generated on two boards, and the final design will feature a board for readout electronics as well. In this proceeding, we investigate the performance of the D-Egg using several existing reconstruction methods developed for IceCube and compare the results against the benchmark PDOM performance.

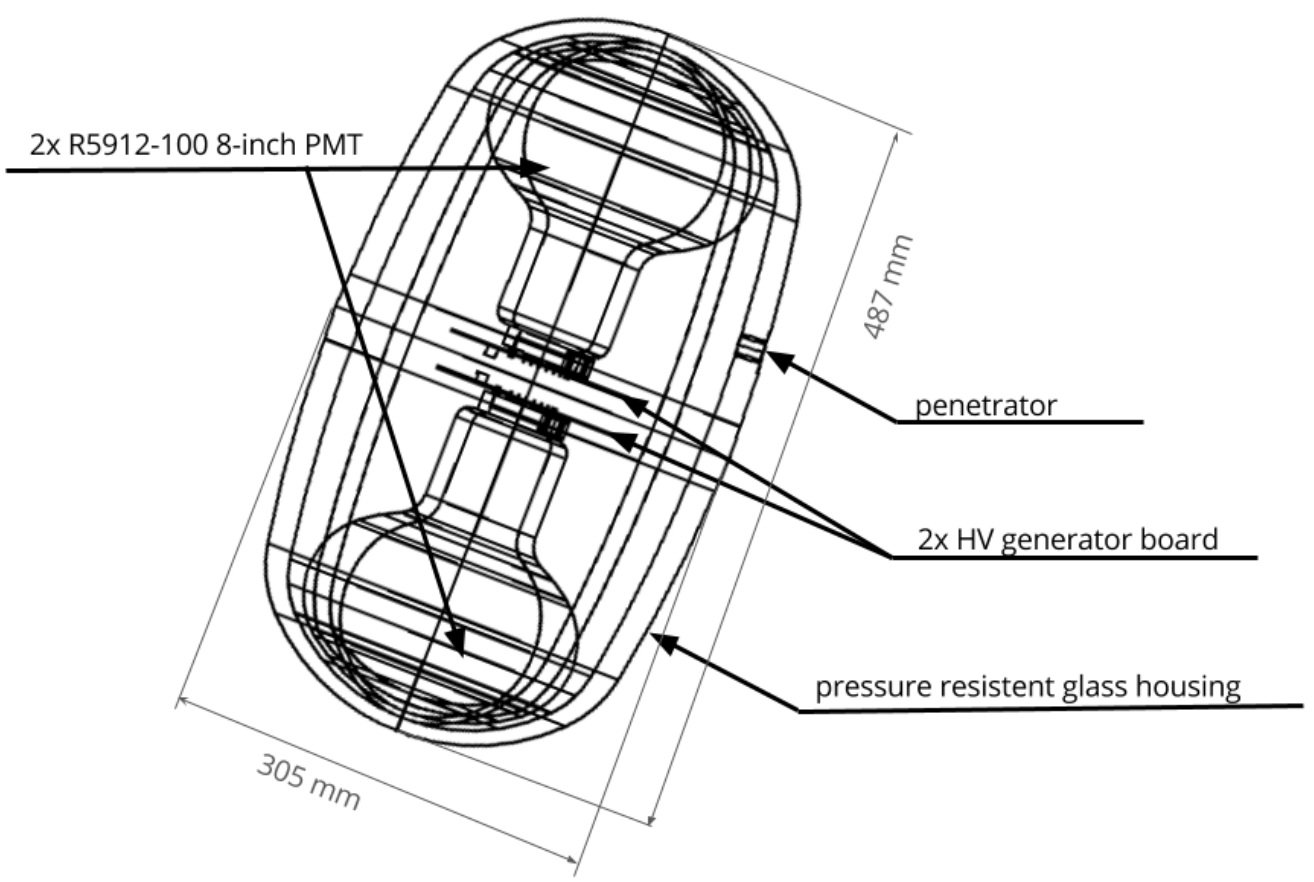

Figure 2: A schematic of the D-Egg design. It features two 8" PMTs enclosed in a highly transparent glass housing, Its diameter is $10 \%$ smaller then that of the current IceCube optical module.

\section{Simulation}

We simulated muons from an $E^{-1.4}$ power-law spectrum in the energy range of $10 \mathrm{TeV}$ to 10 $\mathrm{PeV}$ with a full $4 \pi$ angular distribution. The muons were injected at a cylindrical surface enclosing the detector and then propagated through the ice. The light emerging by stochastic energy losses of the muons as well as the smooth Cherenkov light were simulated and the photon propagation is handled by the direct propagation code developed for IceCube. The simulation features a bulk ice model which means that the ice is homogenous throughout the detector. As the direct propagation of photons is computationally consumptive, the detector simulation for D-Egg and PDOM are sharing the same photon simulation as input, which means that the photons have been simulated once and then the simulation branches into the different types of detector simulation. To further 
increase the simulation efficiency, several simplifications were made. The effects of glass and gel and the module geometry are not simulated individually. Instead the photons are weighted with the angular sensitivity of the module as well as the wavelength dependent quantum efficiency. The efficiency of the photocathode is assumed to be constant over the whole area. To further increase the efficiency of the simulation, the size of the modules is scaled up and the number of propagated photons is decreased accordingly.

The noise introduced by the PMT and the glass housing is simulated in the same way for D-Egg and PDOM, however with absolute values scaled by the photocathode area. Further simplifications are made in the PMT and sensor simulation. The PMT simulation is done as for the PMT used in IceCube, as they are very similar in their behavior. The benefit of this is that the same simulation chain can be used for D-Egg as well as for the IceCube DOM and PDOM. As the readout electronics for the D-Egg is not yet finalized, we assume a perfect readout with an infinitesimal small binning in time. The IceCube array, as part of IceCube-Gen2 has been simulated to our best knowledge.

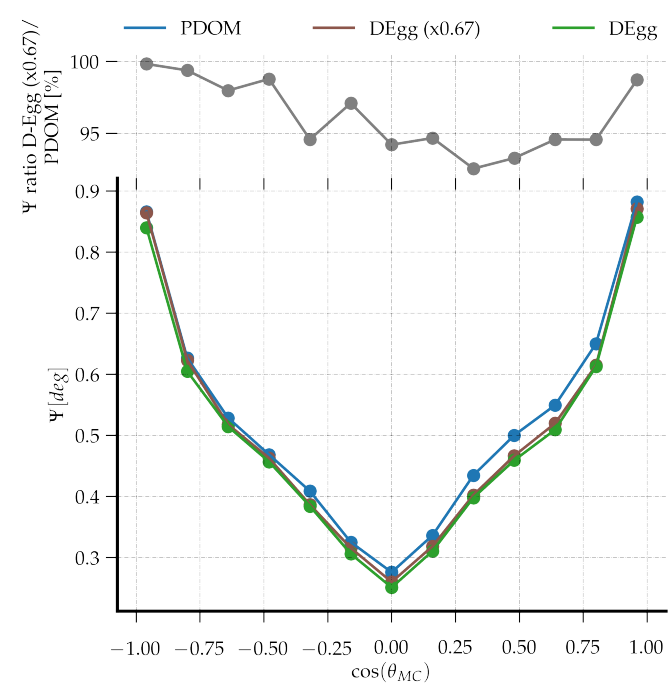

Figure 3: The results of the SPEFit reconstruction for both sensors, D-Egg and PDOM binned in the cosine of the simulated muon direction. The D-Egg effective area is scaled down by a factor of 0.67 to match the PDOM effective area. Muons with a cosine of -1 are entering the detector from below, those with 1 from above respectively.

\section{Muon reconstruction}

The simulated dataset was reconstructed with a set of algorithms. In this study we focus on the reconstruction algorithms SPEFIT and SPLINE-RECO [8]. The algorithms operate on the reconstructed pulses, each using a different method. While SPEFIT uses a simple analytical icemodel and a likelihood with one term per optical module, where only the first registered pulse is considered, SPLINE-RECO is capable of constructing a likelihood with a probability density function (pdf) obtained from tabulated values, and thus is able to also include more complicated models for the glacial ice. To compare the accuracy of the reconstruction results, we looked at the distributions of the opening angle $\Psi$ between the simulated and reconstructed track. The median of this distribution is used as a figure of merit. No quality cuts have been applied, yet we restrict ourself to tracks which traverse the instrumented volume.

We aim to investigate the impact of the increased photocathode area and segmentation on the reconstruction independently. As such, we work with different types of D-Egg simulation: 
- Simulation of the D-Egg "as is" as described in section 3.

- The same as above, however the effective photocathode area is scaled down by a factor of 0.67 to match the photocathode area of the PDOM

- Simulation of the D-Egg where either the upward or downward facing PMT is disabled.

All types of simulations share the same simulated photons, but then branch in different detector simulations. First, the behavior of the two individual PMTs is studied. As the simulation has updown symmetry, we expect the same performance for the datasets with only pulses in the upper or lower PMT. The results for the SPEFIT reconstructions is shown in Figure 3. All reconstructions perform best for more horizontal events due to the fact that the Gen2 geometry is elongated more in the $\mathrm{x}$ and $\mathrm{y}$ dimension than in the $\mathrm{z}$ dimension. This means that horizontal tracks cross a larger instrumented volume. Also as the string spacing is $240 \mathrm{~m}$, vertical tracks have a lower light yield if they enter the detector in between strings. For upward-going muons, if only the lower PMT of D-Egg is used as reconstruction input, it can be seen that the performance is slightly better than for the upper PMT only, and vice versa for downward going muons. The SPEFIT reconstruction yields a higher accuracy for the D-Egg sensor, which we quantify to be about $5 \%$ in the horizontal and downward region due to the segmentation of the D-Egg only as we here compare to the scaleddown version. We attribute this to the fact that SPEFit uses only the first pulse recorded by each PMT, and the doubling of PMT thus increases the number of pulses available to the reconstruction, especially for the downward region.

The performance SPLINE-RECO of the reconstruction is shown in Figure 4. The D-Egg exhibits up to $15 \%$ higher accuracy in reconstruction especially in the horizontal region, which is important to point source searches [4]. The reconstruction in the downward going region yields more accurate results with D-Egg as well. Comparing the results as a function of the true muon energy $E_{M C}$ as it is shown in the right panel of Figure 4, the SPLINE-RECO reconstruction gains for D-Egg due to the higher photoelectron (PE) yield. However it seems that most of the gain results from the larger photcathode area of D-Egg.

\section{Likelihood improvements for segmented sensors}

Figure 4 shows that the increase in reconstruction performance for the D-Egg seems to be attributed mostly to its larger total photocathode area. Thus, we investigate the details of the SPLINE-RECO reconstruction. Developed for IceCube, the here used likelihood is not optimized for segmented sensors, and thus it does not exploit their full potential. The likelihood is given by [8]:

$$
L=\prod_{j=1}^{N_{D O M}} N \cdot p_{j}\left(t_{j}\right) \cdot\left(1-P_{j}\left(t_{1}\right)\right)^{N-1}
$$

In the above equation, $N$ stands for the number of hits on a certain optical module, $p_{j}$ and $P_{j}$ are the time residual pdf and cumulative density function for the hit DOM and $t_{j}$ is the time of the first hit of the given DOM. Contours of this likelihood function can be seen in Figure 5. This simplified example illustrates the likelihood space for a single module, placed in the middle of the individual figures. A muon track crosses the plane of the figure orthogonal in $120 \mathrm{~m}$ distance. In 

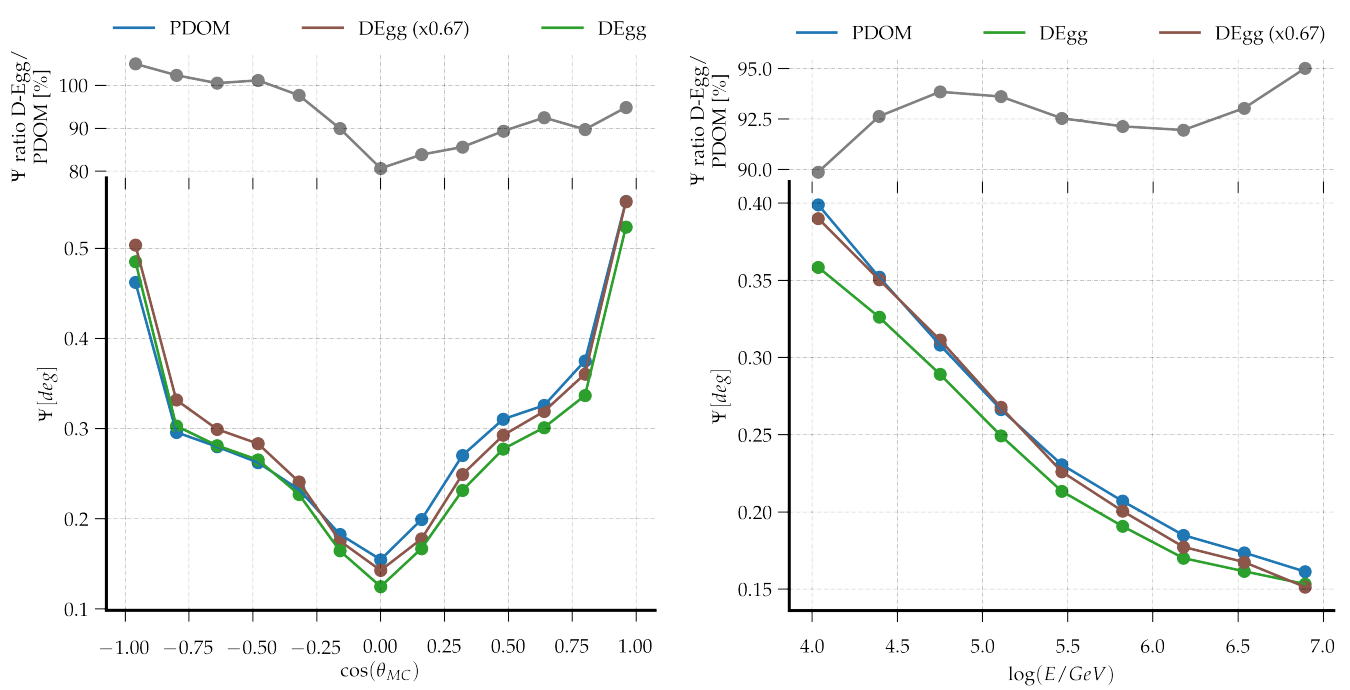

Figure 4: The results of the reconstruction SPLINE-RECO, binned in the cosine of the simulated muon direction on the left and binned in the logarithm of the muon energy on the right. Muons with a cosine of -1 are entering the detector from below, those with 1 from above respectively.

this example, the sensor detects 20 photo electrons with arrival times given by the time residual pdf. The $1 \sigma$ contour for the likelihood developed for the IceCube DOM is shown with the two circles with the red color. As it can be seen, the direction of the individual PMT imposes only very small constraints on the red likelihood contour. As a reason, we suspect the importance of the late photo electrons in the arrival time distribution. In the current approach, the likelihood is constructed by the use of the timing of the first photo electron and the number of total hits, but does not account for the timing of later photo electrons. Extending equation 1 to include the arrival times of all photo electrons can improve the likelihood. This new likelihood is illustrated in the example in Figure 5 with the blue contours. It allows for a more precise determination of the track position, which in total will most likely lead to a better angular resolution of the overall reconstruction algorithm. The IceCube-Gen 2 collaboration is currently working on a reconstruction implementing this approach.

\section{Veto performance}

An effective method to select an all flavor neutrino sample with high purity and full sky acceptance is the implementation of a veto: Using the outer strings and top and bottom layer of optical modules, incoming muon tracks can be tagged and removed from such a sample. The method has been proven successful and lead to the discovery of the extraterrestrial neutrino flux [2].

The method has been applied and studied for IceCube-Gen2 [9]. In the context of this proceedings we are investigating the impact of D-Egg on the efficiency of the veto algorithm. An important parameter of the current algorithm is the veto threshold, which is the charge required in the veto region to trigger the veto, which is currently set to 3 PEs. Since the D-Egg has an upward facing PMT, we expect a higher performance for downward going cosmic ray muon tracks. Due to the D-Eggs larger photo cathode, we also expect a higher probability to detect charge in the veto region 


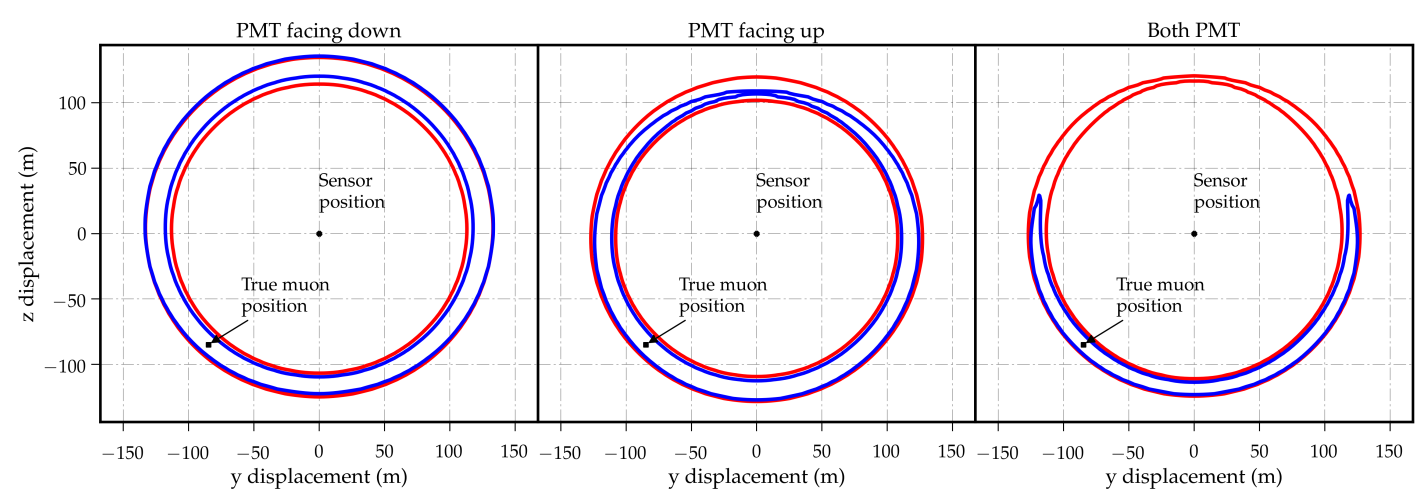

Figure 5: Likelihood contours of two different likelihoods for a single D-Egg sensor in case of a muon traversing the plane in orthogonal direction. The red contour results from the likelihood used in SPLINERECO, the blue contour is a proposed likelihood considering the timting of the late pulses in the arrival time distribuiton. On the left, the contours are shown for the lower PMT only. The contours are base on an Asimove sample. The middle plot shows the situation for the upper PMT and on the right the combined contours of both PMTs are shown.

at all. This is illustrated in Figure 6: The distribution of collected charge for the upper 2 layers of modules of the IceCube-Gen2 geometry results in a higher probabilty to veto incoming muons. Further impact of the use of D-Eggs in the veto region is currently under investigation.

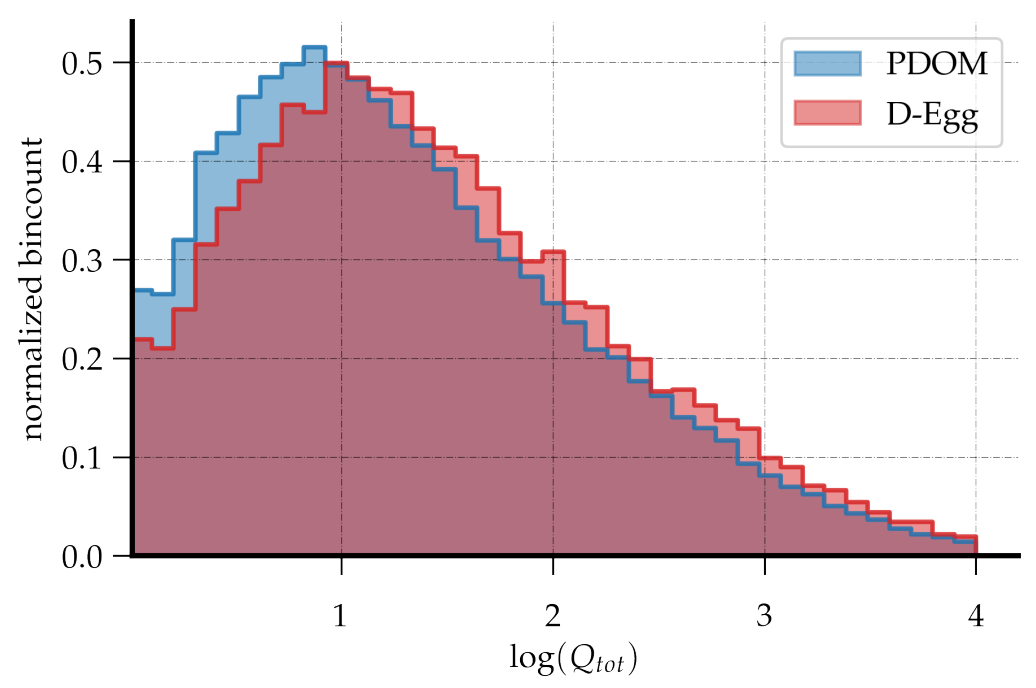

Figure 6: The collected charge in the upper layer of the IceCube-Gen2 array. The collected charge is shown for the uppermost 2 layers of optical modules.

\section{Summary}

For the first time, we present a study of muon track angular resolutions with current reconstruction techniques used by IceCube for the proposed extension IceCube-Gen2. We compare a new sensor design, the D-Egg, to an improved sensor based on the current IceCube design (PDOM). The angular resolution for common reconstruction algorithms in IceCube were studied for both sensors with a full-sky muon simulation and we find an improvement of the angular resolution of 
D-Egg of about $20 \%$ compared to PDOM. The major part of the improvement can be attributed to the larger photo cathode area of D-Egg. Investigating the fact that segmentation of the sensors seems to have only a small impact on the reconstruction result, we find that there are no existing reconstruction methods that fully take advantage of the module segmentation. Efforts are ongoing to develop a reconstruction that more accurately incorporates late photon timing information, and thus exploits all of the information provided by the D-Egg module. Besides the improvement in angular resolution, we show that the veto performance for the current implementation of the IceCube veto can be improved by using D-Eggs as well. We studied the deposited charge in the upper layer of the IceCube-Gen2 array and find a significant increase in the low charge region around the $3 \mathrm{PE}$ threshhold. In conclusion, we find that we are on a good track to improve the current IceCube reconstruction and veto techniques to exploit the full potential of new approaches in sensor design for IceCube-Gen2 and encourage further, more detailed studies to follow.

\section{References}

[1] IceCube Collaboration, Aartsen et al., JINST 12 (2017), P03012

[2] IceCube Collaboration, M.G. Aartsen et. al., SCI 342(6161) (2006) P1242856.

[3] IceCube Collaboration, M. G. Aartsen et al., astro-ph/1412. 5106 (2014).

[4] IceCube-Gen2 Collaboration, PoS ( ICRC2017) 991 (these proceedings).

[5] IceCube-PINGU Collaboration, P. Sandstrom, AIP 1630(1) (2014) P180-183.

[6] K. Hanson and O. Tarasova, NIM 567(1) (2006) P214-217

[7] IceCube-Gen2 Collaboration, PoS ( ICRC2017) 1051 (these proceedings).

[8] AMANDA Collaboration, J. Ahrens et al., NIMA524 (2004) P169-194.

[9] IceCube-Gen2 Collaboration, POS ( ICRC2 017) 945 (these proceedings). 